\title{
TIDAL EVOLUTION OF RUBBLE PILES
}

\author{
Peter Goldreich ${ }^{1,2}$ AND Re'Em SARI ${ }^{2,3}$ \\ ${ }^{1}$ Institute for Advanced Study, Princeton, NJ, USA \\ ${ }^{2}$ Caltech 130-33, Pasadena, CA 91125, USA \\ ${ }^{3}$ Racah Institute of Physics, Hebrew University, Jerusalem 91904, Israel \\ Received 2007 December 4; accepted 2008 September 15; published 2009 January 7
}

\begin{abstract}
Many small bodies in the solar system are believed to be rubble piles, a collection of smaller elements separated by voids. We propose a model for the structure of a self-gravitating rubble pile. Static friction prevents its elements from sliding relative to each other. Stresses are concentrated around points of contact between individual elements. The effective dimensionless rigidity, $\tilde{\mu}_{\text {rubble }}$, is related to that of a monolithic body of similar composition and size, $\tilde{\mu}$ by $\tilde{\mu}_{\text {rubble }} \sim \tilde{\mu}^{1 / 2} \epsilon_{Y}^{-1 / 2}$, where $\epsilon_{Y} \sim 10^{-2}$ is the yield strain. This represents a reduction in effective rigidity below the maximum radius, $R_{\max } \sim\left[\mu \epsilon_{Y} /\left(G \rho^{2}\right)\right]^{1 / 2} \sim 10^{3} \mathrm{~km}$, at which a rubble pile can exist. Our model for the rigidity of rubble piles is compatible with laboratory experiments on the speed of shear waves in sand. Densities derived for binary asteroids imply that they are rubble piles. Thus their tidal evolution proceeds faster than it would if they were monoliths. Binary orbit evolution is also driven by torques resulting from the asymmetrical scattering and reradiation of sunlight (YORP and BYORP effects). The tidal torque probably overcomes the radiative (YORP) torque and synchronizes the spins of secondaries in near-Earth binary asteroids and it definitely does so for secondaries of main-belt binary asteroids. Synchronization is a requirement for the radiative (BYORP) torque to act on the binary orbit. This torque clearly dominates the tidal torque for all near-Earth binary asteroids and for some binaries in the main belt. For other main-belt binaries, the tidal torque appears to be at least comparable in strength to the BYORP torque. An exciting possibility is that in these systems the angular momentum added to the orbit by the tidal torque might be removed by the radiative torque.
\end{abstract}

Key words: minor planets, asteroids - solar system: general

Online-only material: color figure

\section{INTRODUCTION}

Rubble piles are bodies composed of smaller elements separated by voids. There is compelling evidence that some small solar system bodies are rubble piles bound by gravity. Their telltale signature is a mean density below that of their constituent elements. The examples include four icy satellites of Saturn, the co-orbitals, Janus and Epimetheus, and the F-ring shepherds, Prometheus and Pandora (Jacobson \& French 2004; Porco et al. 2007), the rocky main-belt asteroids, C-type 253 Mathilde (Veverka et al. 1997; Yeomans et al. 1997) and M-type 22 Kalliope (Margot \& Brown 2003), and the binary near-Earth asteroid 1999 KW4 (Ostro et al. 2006). The largest of these bodies, Janus and Kalliope, have dimensions of order $100 \mathrm{~km}$. It is unclear whether larger rubble piles exist, but it is clear that some small bodies are not rubble piles. A significant fraction of asteroids with diameters below $150 \mathrm{~m}$ spin so fast that they must be bound by material strength rather than gravity. Perhaps larger asteroids that are rubble piles contain elements of order $150 \mathrm{~m}$ in size.

Intuitively, we expect a rubble pile to be weaker than a monolithic body of the same composition. In what follows, we provide a theoretical basis for estimating tidal evolution rates in binary rubble piles. Our paper is organized as follows. In Section 2, we formulate a quantitative theory for the effective dimensionless rigidity of a self-gravitating rubble pile and demonstrate that it is due to voids rather than cracks. Limits on the sizes of rubble piles are derived in Section 3. Implications of our theory for the tidal evolution of binary rubble piles are described in Section 4. Then in Section 5, we compare the relative rates of binary evolution due to tidal torques with that due to torques arising from the asymmetrical scattering, absorption, and re-emission of solar radiation (YORP and BYORP).

\section{EFFECTIVE ELASTIC MODULUS OF A RUBBLE PILE}

We begin by reviewing the tidal response of a uniform body of density $\rho$, rigidity $\mu$, and radius $R$. As is customary, we define the dimensionless rigidity by $\tilde{\mu}$,

$$
\tilde{\mu} \equiv \frac{19 \mu}{2 g \rho R} \text {. }
$$

Next, we show that $\tilde{\mu}$ is the ratio of the fluid strain to the elastic strain. ${ }^{4}$

We assume that the tidal force, $f$, is weak in comparison to the cohesive force of the body's self-gravity, $g M$, where $g \sim G \rho R$. If the body were fluid, $\mu=0$, it would suffer a strain

$$
\epsilon_{g} \sim \frac{f}{g \rho R^{3}},
$$

whereas if it were elastic but lacked self-gravity, $g=0$, the strain would be

$$
\epsilon_{\mu} \sim \frac{f}{\mu R^{2}} .
$$

To order of magnitude, the ratio between Equations (2) and (3) reproduces $\tilde{\mu}$ given by Equation (1).

How does the tidal response of a rubble pile differ from that of a monolith? To answer this question, we investigate some simple models.

\footnotetext{
4 Arguments in this section are order of magnitude only.
} 


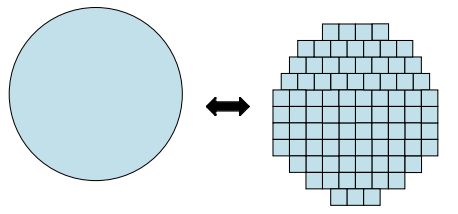

$$
\tilde{\mu}=\frac{19}{2} \frac{\mu}{\rho R g}
$$$$
\text { no sliding }
$$

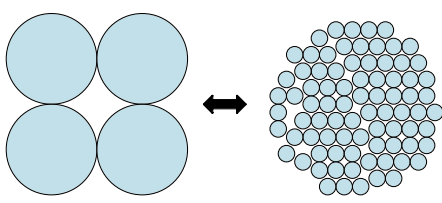

$$
\tilde{\mu}_{\text {spheres }} \sim \tilde{\mu}^{2 / 3}
$$$$
R<10 \mathrm{~km}
$$

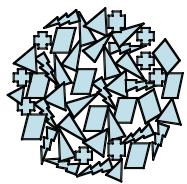

$$
\begin{gathered}
\tilde{\mu}_{\text {rubble }} \sim\left(\tilde{\mu} / \epsilon_{Y}\right)^{1 / 2} \\
R<1000 \mathrm{~km}
\end{gathered}
$$

Figure 1. Three simple models of fragmented bodies. Upper row depicts a body composed of cubical elements. There are no voids. Static friction prevents the elements from sliding relative to each other. Its effective dimensionless rigidity is identical to that of a monolith. Middle row shows a body composed of spherical elements. Voids are present. Stresses concentrate near contacts between elements. The effective dimensionless rigidity is smaller than that of a monolith and is independent of the sizes of the elements. Such an idealized configuration requires the spheres to be sufficiently smooth. If made of rock or ice, its radius could not be larger than about $10 \mathrm{~km}$. Bottom row illustrates a more realistic rubble pile composed of irregular elements. Sharper contact points increase stress concentration more than for a body composed of spherical elements. Accordingly, its effective dimensionless rigidity is further decreased. Radii of rubble piles composed of rock or ice cannot be larger than about $1000 \mathrm{~km}$.

(A color version of this figure is available in the online journal.)

\subsection{Cracks Do Not Matter}

Normal stresses are seamlessly transmitted across cracks, so a body's response to weak tides is unaffected by cracks. Consider a body of radius $R$ composed of cubical elements whose sides have length $r \ll R$ (see upper row of Figure 1). The ratio of the weight of a single cube, $g M(r / R)^{3}$, to the divergence of tidal stress acting on its volume, $f(r / R)^{3}$, is just $g M / f$. Thus a coefficient of static friction larger than $f / g M \sim\left(R^{\prime} / a\right)^{3}$, where $R^{\prime}$ is the radius of the tide raising body and $a$ is the binary's semimajor axis, ${ }^{5}$ would suffice to prevent the cubes from slipping relative to each other. Since coefficients of static friction for rocks and dry ice are of order unity, except for the tightest binaries, slipping would not occur.

\subsection{Voids are Key}

\subsubsection{Uniform Spheres}

Next, we consider a body of radius $R$ composed of identical spheres of radius $r \ll R$ (see middle row of Figure 1). Its mean density $\bar{\rho} \approx 0.7 \rho$. A typical cross section cuts $(R / r)^{2}$ small spheres each of which transmits forces $F(r / R)^{2}$ to its neighbors, where $F \sim g M+f$ includes both tidal forces and self-gravity. In doing so, each small sphere undergoes a linear distortion $\delta x$ and forms contact surfaces with its neighbors of area $\delta x r$. Within $(\delta x r)^{1 / 2}$ of each contact, the strain is of order $(\delta x / r)^{1 / 2}$ so

$$
F\left(\frac{r}{R}\right)^{2} \sim \mu r^{1 / 2} \delta x^{3 / 2}
$$

\footnotetext{
5 We are assuming identical densities for both members of the binary.
}

The average strain is just $\delta x / r$, where from Equation (4)

$$
\frac{\delta x}{r} \sim\left(\frac{F}{\mu R^{2}}\right)^{2 / 3} .
$$

Most of this strain is due to the body's self-gravity. To isolate the tidal strain, we expand $F^{2 / 3}$ in Equation (5) around $F \sim g M$ to obtain

$$
\epsilon \sim \frac{f}{\mu R^{2}}\left(\frac{\mu}{g \rho R}\right)^{1 / 3} .
$$

Thus the effective dimensionless tidal rigidity of a body composed of identical spheres is

$$
\tilde{\mu}_{\text {spheres }} \sim\left(\frac{\mu}{g \rho R}\right)^{1 / 3} \sim \tilde{\mu}^{2 / 3} .
$$

This result is an extension to self-gravitating rubble piles of a model originally developed by Duffy \& Mindlin (1957).

\subsubsection{Irregular Fragments}

Natural rubble piles are likely to be composed of irregularly shaped elements whose surfaces have local radii of curvature, $\hat{r}$, that are much smaller than the elements' sizes, $r$ (see bottom row of Figure 1). Compared to rubble piles composes of spheres, contact areas would be reduced, stress concentrations increased, and the effective dimensionless rigidity lowered. A simple modification of the derivation given in Section 2.2.1 suffices to evaluate the effective dimensionless rigidity of a rubble pile, $\tilde{\mu}_{\text {rubble }}$. Each element still transmits its share of the total force. However, $\hat{r}$ must replace $r$ on the right-hand side of Equation (4). Thus now

$$
\frac{\delta x}{r} \sim\left(\frac{F}{\mu R^{2}}\right)^{2 / 3}\left(\frac{r}{\hat{r}}\right)^{1 / 3} .
$$

Continuing as before, we find that the average strain across the rubble pile is increased by the factor $(r / \hat{r})^{1 / 3}$ with the consequence that the effective dimensionless rigidity now reads

$$
\tilde{\mu}_{\text {rubble }} \sim \tilde{\mu}_{\text {spheres }}\left(\frac{\hat{r}}{r}\right)^{1 / 3} \sim \tilde{\mu}^{2 / 3}\left(\frac{\hat{r}}{r}\right)^{1 / 3} .
$$

The sharper the contact points, the softer the rubble pile, up to a limit at which the stress near the contact surfaces reaches the material's yield stress $\sigma_{Y}$, or yield strain $\epsilon_{Y}=\sigma_{Y} / \mu$. This limit is met at

$$
\frac{\hat{r}}{r} \sim \frac{1}{\left(\tilde{\mu} \epsilon_{Y}^{3}\right)^{1 / 2}} .
$$

Sharper contact points than allowed by Equation (10) would be dulled by material flow or failure. Therefore,

$$
\tilde{\mu}_{\text {rubble }} \gtrsim\left(\frac{\tilde{\mu}}{\epsilon_{Y}}\right)^{1 / 2} .
$$

Equation (9) demonstrates that the effective dimensionless rigidity of a rubble pile, $\mu_{\text {rubble }}$, is smaller than that of a monolithic body of the same size. The reduction of $\mu_{\text {rubble }}$ arises from the concentration of stresses due to the presence of voids. 


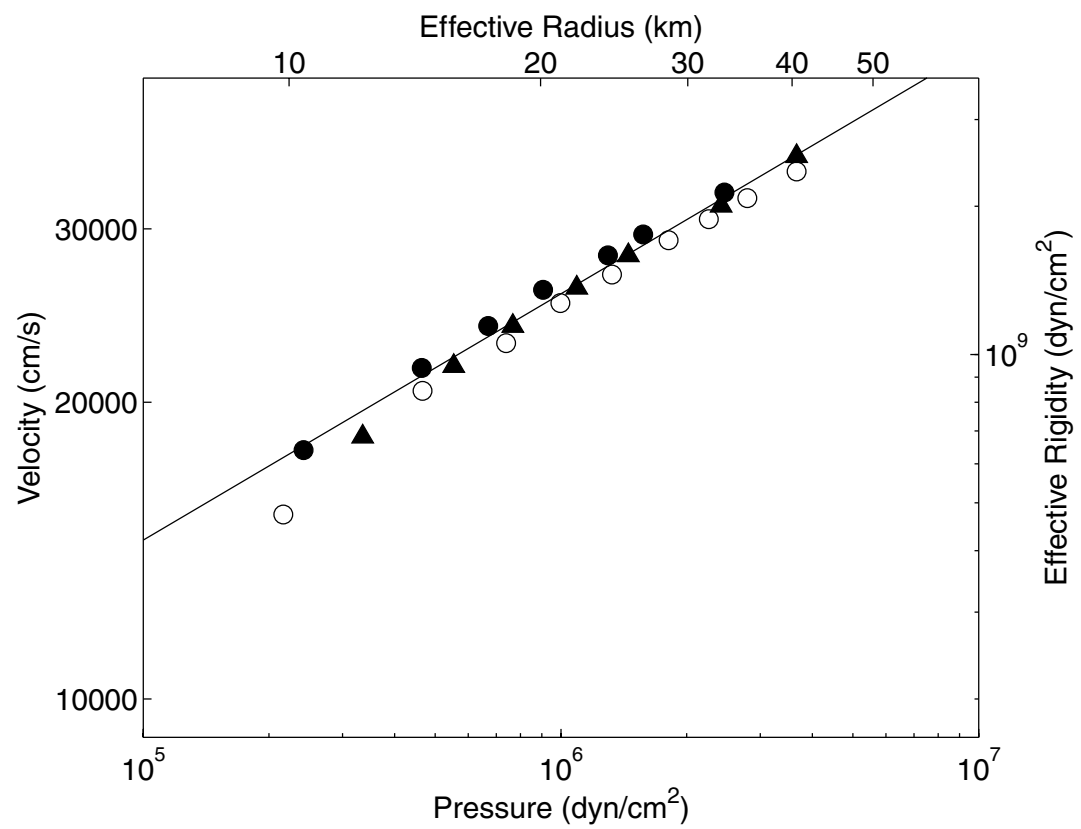

Figure 2. Comparison of our model for the effective rigidity of rubble piles with that from experiments on sand taken from Goddard (1990). Shear wave velocity as a function of pressure in saturated, dry, and drained Ottawa sands is shown by open circles, solid circles, and triangles, respectively. The upper abscissa indicates the radius of a rocky rubble pile whose central pressure corresponds to that given on the lower abscissa.

\subsection{Energy Considerations}

We rederive Equation (9) based on energy considerations. Strains of order $(\delta x / \hat{r})^{1 / 2}$ are attained within a distance $(\delta x \hat{r})^{1 / 2}$ of the contacts between individual elements. Thus the elastic energy stored within the rubble pile satisfies

$$
\delta E \sim \mu(\delta x)^{5 / 2} \hat{r}^{1 / 2}\left(\frac{R}{r}\right)^{3} .
$$

We can also express $\delta E$ in terms of the effective dimensionless rigidity, $\tilde{\mu}_{\text {rubble }}$ and the average strain in the rubble pile, $\delta x / r$, as

$$
\delta E \sim \tilde{\mu}_{\text {rubble }} g \rho R\left(\frac{\delta x}{r}\right)^{2} R^{3} .
$$

Equating the expressions for $\delta E$ given in Equations (12) and (13), we arrive at

$$
\tilde{\mu}_{\text {rubble }} \sim \tilde{\mu} \frac{\delta x^{1 / 2} \hat{r}^{1 / 2}}{r} .
$$

Finally, by using Equation (8) to eliminate $\delta x$, we recover Equation (9).

\subsection{Comparison with Experiments}

For comparison with experiment, it is more convenient to work in terms of the effective dimensional rigidity, $\mu_{\text {rubble }}$. From Equations (1) and (11), it follows that the limiting value of $\mu_{\text {rubble }} \gtrsim\left(g \rho R \mu / \epsilon_{Y}\right)^{1 / 2}$. Experimentally, it is generally found that the effective rigidity of a granular material scales in direct proportion to the square root of the confining pressure. The original explanation for this scaling law is by Goddard (1990). Our contribution is its application to self-gravitating bodies.

Laboratory measurements of the shear velocity, $c_{s}=\sqrt{\mu / \rho}$, in sand as a function of pressure provide a useful calibration. The data on $c_{s}(p)$ plotted in Figure 1 of Goddard (1990) are replotted in our Figure 2. The right-hand boundary shows the effective rigidity corresponding to the shear velocity. The points are well-fit by the solid line that is derived from our expression for effective rigidity with $\epsilon_{Y} \cong 0.17$. $^{6}$ This should not be taken as evidence that the yield strain of sand is 0.17 since our formula is only accurate to order of magnitude.

On the upper boundary of Figure 2 we display the radius of an asteroid with $\rho \cong 2 \mathrm{~g} \mathrm{~cm}^{-3}$, whose average internal pressure

$$
P=(4 \pi / 15) G \rho^{2} R^{2} \cong 2.2 \times 10^{3}\left(\frac{R}{1 \mathrm{~km}}\right)^{2} \text { dyne } \mathrm{cm}^{-2},
$$

corresponds to that given on the lower boundary. The range of pressures covered in the experiments on sand correspond to those inside asteroids with radii from 10 to $40 \mathrm{~km}$.

\section{CRITICAL SIZES FOR RUBBLE PILES}

\subsection{Mechanical Limits}

We would expect the voids in rubble piles to occupy a decreasing fraction of the volume with increasing $R$.

At

$$
R=R_{*} \sim\left(\frac{\mu \epsilon_{Y}^{3}}{\rho^{2} G}\right)^{1 / 2},
$$

we find $\hat{r} / r \sim 1$ and therefore $\tilde{\mu}_{\text {rubble }} \sim \tilde{\mu}_{\text {spheres. Therefore, a }}$ body composed of identical spheres would avoid elastic failure provided $R<R_{*}$. For $R>R_{*}$, elastic failure would occur at points of contact among spheres. The effective dimensionless rigidity for $R>R_{*}$ would thus be given by Equation (11) rather than by Equation (7) even if the body was assembled from spheres. A rubble pile with $R=R_{*}$ would have $\tilde{\mu} \sim \epsilon_{Y}^{-2}$ as compared to the much larger value $\tilde{\mu}_{\text {rubble }} \sim \epsilon_{Y}^{-3}$ for a monolith of similar size. Adopting nominal values of $\epsilon_{Y} \sim 10^{-2}, \rho_{\text {rock }} \approx$ $3.5 \mathrm{~g} \mathrm{~cm}^{-3}, \mu_{\text {rock }} \approx 5 \times 10^{11}$ dyne $\mathrm{cm}^{-2}, \rho_{\text {ice }} \approx 1 \mathrm{~g} \mathrm{~cm}^{-3}$, and

6 The dashed line indicates the higher effective rigidity of a body composed of uniform quartz spheres. 
$\mu_{\text {ice }} \approx 3 \times 10^{10}$ dyne $\mathrm{cm}^{-2}$, we obtain $R_{*} \sim 10 \mathrm{~km}$ for rubble piles composed of either rock or ice. Moreover, $\tilde{\mu}_{\text {rubble }} \sim 10^{4}$ as compared to $\tilde{\mu} \sim 10^{6}$.

At

$$
R_{\max }=\left(\frac{\mu \epsilon_{Y}}{\rho^{2} G}\right)^{1 / 2},
$$

the average interior pressure $g \rho R_{\max } \sim \sigma_{Y}$, so voids could only exist near the surface. Areas of contact are comparable to surface areas of individual elements, $\hat{r} / r \sim \epsilon_{Y}^{-2}$, and $\tilde{\mu}_{\text {rubble }} \sim \tilde{\mu} \sim \epsilon_{Y}^{-1}$. With our nominal parameters, $R_{\max } \sim$ $10^{3} \mathrm{~km}$ and $\tilde{\mu}_{\text {rubble }} \sim \tilde{\mu} \sim 10^{2}$.

\subsection{Thermal Limits}

Rubble piles should be more common among smaller bodies because they cool more rapidly than larger ones and therefore are less likely to have been melted. Thermal diffusivities of rock and ice are of order $10^{-2} \mathrm{~cm}^{2} \mathrm{~s}^{-1}$, which implies

$$
t_{\text {cool }} \sim 3 \times 10^{10}\left(\frac{R}{10^{3} \mathrm{~km}}\right)^{2} \mathrm{y} .
$$

Even bodies as small as $R_{*} \sim 10 \mathrm{~km}$ might have been melted if they formed early and were endowed with shortlived radioactive nuclides. On the other hand, bodies as large as $R_{\text {max }} \sim 3 \times 10^{2} \mathrm{~km}$, which were fragmented by collisions after the short-lived radioactive nuclides had decayed, could have avoided melting.

\section{IMPLICATIONS FOR TIDAL EVOLUTION}

Tidal friction may play a crucial role in the orbital and spin evolution of binary asteroids. We consider tides raised on both primary, $p$, and secondary, $s$, under the assumption that the two bodies are composed of identical material. Evolution rates depend on two parameters, the tidal Love number, $k$, and the tidal quality factor, $Q$ (Murray \& Dermott 2000). ${ }^{7}$

Tides raised by the primary on the secondary drive the latter's spin, $\omega_{s}$, toward synchronization with the mean orbital angular velocity, $n$, according to

$$
\frac{d \omega_{s}}{d t}=5 \pi \operatorname{sgn}\left(n-\omega_{s}\right) \frac{k_{s}}{Q_{s}} G \rho_{p}\left(\frac{\rho_{p}}{\rho_{s}}\right)\left(\frac{R_{p}}{a}\right)^{6} .
$$

This is the fastest process driven by tides. Its rate may be expressed by dividing Equation (19) by the breakup spin rate $\bar{\omega}_{s} \equiv\left(G M_{s} / R_{s}^{3}\right)^{1 / 2}$,

$$
\frac{1}{\bar{\omega}_{s}} \frac{d \omega_{s}}{d t}=\frac{15}{4} \operatorname{sgn}\left(n-\omega_{s}\right) \frac{k_{s}}{Q_{s}}\left(\frac{\rho_{p}}{\rho_{s}}\right)^{3 / 2}\left(\frac{R_{p}}{a}\right)^{9 / 2} n
$$

Next, we focus on tidal evolution after the secondary's spin has become synchronous with the mean orbital angular velocity while the primary's spin remains much faster. In this case, tides raised on the primary cause both the semimajor axis, $a$, and the orbital eccentricity, $e$, to grow while those raised on the secondary have negligible effect on the semimajor axis and cause the eccentricity to decay (Goldreich 1963; Goldreich \& Soter 1966). Relevant expressions for $e \ll 1$ are

$$
\frac{1}{a} \frac{d a}{d t}=3 \frac{k_{p}}{Q_{p}} \frac{M_{s}}{M_{p}}\left(\frac{R_{p}}{a}\right)^{5} n
$$

\footnotetext{
$7 Q^{-1}$ is a stand-in for $\sin 2 \delta$, where $\delta$ is the tidal phase lag.
}

and

$$
\frac{1}{e} \frac{d e}{d t}=\frac{57}{8} \frac{k_{p}}{Q_{p}} \frac{M_{s}}{M_{p}}\left(\frac{R_{p}}{a}\right)^{5} n,
$$

for tides raised on the primary, and

$$
\frac{1}{e} \frac{d e}{d t}=-\frac{21}{2} \frac{k_{s}}{Q_{s}} \frac{M_{p}}{M_{s}}\left(\frac{R_{s}}{a}\right)^{5} n,
$$

for tides raised on the secondary.

The tidal Love number for a uniform monolith is given by

$$
k=\frac{1.5}{1+\tilde{\mu}} .
$$

Scaling from the measured tidal Love number of the Moon, $k_{\text {Moon }} \approx 0.025$ (Williams et al. 2008), which corresponds to

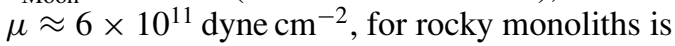

$$
\tilde{\mu} \approx 2 \times 10^{8}\left(\frac{\mathrm{km}}{R}\right)^{2} .
$$

Thus from Equation (11) with $\epsilon_{Y}=10^{-2}$, we obtain

$$
k_{\text {rubble }} \lesssim 10^{-5} \frac{R}{\mathrm{~km}} .
$$

The tidal quality factor, $Q$, is more uncertain. Available evidence suggests that $Q \sim 10^{2}$ for monoliths (Goldreich \& Soter 1966). However, $Q$ for a rubble pile may be much smaller because the yield stress is approached near points of contact among its individual elements. This issue could and should be addressed experimentally.

Generally tidal evolution proceeds in a similar fashion but more rapidly in binary rubble piles than in binary monoliths composed of similar material. Eccentricity evolution is an exception. Although eccentricity tends to increase in strengthdominated binary monoliths, it may damp in binary rubble piles.

For strength-dominated bodies, $\tilde{\mu} \gg 1$, the ratio of the rates of eccentricity excitation and damping are

$$
\mathcal{R}=\frac{19}{28}\left(\frac{\rho_{s}}{\rho_{p}}\right)^{2} \frac{R_{s}}{R_{p}} \frac{\tilde{\mu}_{s}}{\tilde{\mu}_{p}} \frac{Q_{s}}{Q_{p}} .
$$

For monoliths of identical composition and $Q,{ }^{8}$ this ratio reduces to

$$
\mathcal{R}_{\text {monolith }}=\frac{19}{28} \frac{R_{p}}{R_{s}},
$$

so eccentricity would increase unless $R_{p} / R_{S}<1.47$, which corresponds to a mass ratio less than 3.20 . For primary and secondary composed of spherical elements with identical compositions and $Q$ 's, the ratio reads

$$
\mathcal{R}_{\text {spheres }}=\frac{19}{28}\left(\frac{R_{p}}{R_{s}}\right)^{1 / 3},
$$

so eccentricity would grow unless $R_{p} / R_{s}<3.2$, which corresponds to a mass ratio below 33 . Finally, for rubble piles composed of irregular elements of identical compositions and $Q$ 's,

$$
\mathcal{R}_{\text {rubble }}=\frac{19}{28},
$$

so eccentricity would damp for all mass ratios.

\footnotetext{
8 Identical compositions do not guarantee identical $Q \mathrm{~s}$, because the latter
} may also depend on strain, strain rate, temperature, and pressure. 
It is clear that eccentricity damping is more likely for binary rubble piles than for binary monoliths. However, given the uncertainties in the relative values of the primary's and secondary's $\tilde{\mu}_{\text {rubble }}$ and $Q$, eccentricity growth remains a possibility, in particular for large mass ratios.

\section{COMPARISON OF RADIATIVE AND TIDAL TORQUES}

In this section, we assess the relative importance of tidal and radiative torques on the evolution of binary near-Earth and main-belt asteroids. General trends are readily discerned but our ability to draw firm conclusions is frustrated by poorly constrained values of crucial parameters. Where needed, we adopt the expression for the tidal Love number given by Equation (26).

\subsection{YORP}

The spin rate of an isolated body changes in response to the asymmetrical scattering and reradiation of the sunlight it intercepts. This is known as the YORP (Yarkovsky-O'KeefeRadzievskii-Paddack) effect (Rubincam 2000). The radiation torque would be at maximum strength if all the intercepted sunlight were absorbed and then re-emitted tangentially from the body's equator either forward or backward with respect to the spin velocity. We parameterize the reduction of the torque relative to this maximum value by a factor $f_{Y} \cdot{ }^{9}$ The rate of change of spin due to YORP then reads

$$
\frac{1}{\bar{\omega}} \frac{d \omega}{d t}=\frac{15 \sqrt{3} f_{Y}}{64 \pi^{3 / 2}} \frac{L_{\odot}}{c G^{1 / 2} \rho^{3 / 2} R^{2} A^{2}},
$$

where $A$ is the distance from the Sun and $L_{\odot}$ is the solar luminosity.

The YORP effect has been verified observationally; Taylor et al. (2007) and Lowry et al. (2007) measure a spin-up rate of $4.7 \times 10^{-16} \mathrm{~s}^{-2}$ for asteroid 2000 PH5. With a mean radius of about $56 \mathrm{~m}$, this implies $f_{Y} \sim 4 \times 10^{-4}$.

For later applications we need the ratio of the tidal and YORP contributions to the rate of change of the satellite's spin. From Equations (20) and (31), their relative magnitudes are

$$
\begin{aligned}
\mathcal{R}_{\dot{\omega}_{s}}= & \frac{32 \pi^{2}}{3\left|f_{Y}\right|} \frac{k_{s} \mid}{Q_{s}} \frac{c G \rho_{p}^{2}}{L_{\odot}} \frac{R_{p}^{6} R_{s}^{2} A^{2}}{a^{6}} \\
\approx & \frac{10^{3}}{\left|f_{Y}\right| Q_{s}}\left(\frac{\rho_{p}}{\mathrm{~g} \mathrm{~cm}^{-3}}\right)^{2}\left(\frac{R_{p}}{\mathrm{~km}}\right)^{6}\left(\frac{R_{s}}{\mathrm{~km}}\right)^{3} \\
& \times\left(\frac{A}{\mathrm{AU}}\right)^{2}\left(\frac{\mathrm{km}}{a}\right)^{6} .
\end{aligned}
$$

\subsection{BYORP}

The orbital angular momentum of a spin-orbit synchronized satellite changes in response to the asymmetrical scattering and reradiation of the sunlight it intercepts. This is known as the binary YORP (BYORP) effect (Ćuk \& Burns 2005). The radiation torque would be maximal if all the intercepted sunlight were absorbed and then re-emitted either forward or backward with respect to the orbital velocity. We parameterize the reduction of the torque relative to the maximal value by a

\footnotetext{
$9 f_{Y}$ can be positive or negative.
}

factor $f_{B Y} \cdot{ }^{10}$ The rate of change of binary semimajor axis due to BYORP then reads

$$
\frac{1}{a} \frac{d a}{d t}=\frac{f_{B Y}}{2} \frac{L_{\odot} R_{s}^{2} a^{1 / 2}}{c\left(G M_{p}\right)^{1 / 2} M_{s} A^{2}} .
$$

Again for later applications we need the ratio of the tidal and YORP contributions to rate of change of the semimajor axis. Its magnitude, given by dividing Equation (21) by Equation (33), reads

$$
\begin{aligned}
\mathcal{R}_{\dot{a}}= & \frac{32 \pi^{2}}{3\left|f_{B Y}\right|} \frac{k_{p}}{Q_{p}} \frac{c G \rho_{s}^{2}}{L_{\odot}} \frac{R_{p}^{5} R_{s}^{4} A^{2}}{a^{7}} \\
\approx & \frac{10^{3}}{\left|f_{B Y}\right| Q_{p}}\left(\frac{\rho_{s}}{\mathrm{~g} \mathrm{~cm}^{-3}}\right)^{2}\left(\frac{R_{p}}{\mathrm{~km}}\right)^{6}\left(\frac{R_{s}}{\mathrm{~km}}\right)^{4} \\
& \times\left(\frac{A}{\mathrm{AU}}\right)^{2}\left(\frac{\mathrm{km}}{a}\right)^{7} .
\end{aligned}
$$

\subsection{Tidal Synchronization as a Precondition for BYORP}

BYORP requires the satellite to spin synchronously. The tidal torque a planet exerts on a satellite drives its spin toward the synchronous value. ${ }^{11}$ Provided the magnitude of the tidal torque exceeds that of the radiative torque, $\mathcal{R}_{\dot{\omega}_{s}}>1$, the satellite will end up spinning synchronously. For $\mathcal{R}_{\dot{\omega}_{s}}<1$, the situation is more complicated. Should the radiative torque drive the spin toward synchroneity, it might be captured in the synchronous resonance. The probability for this to occur is $2 \mathcal{R}_{\dot{\omega}_{s}} /\left(1+\mathcal{R}_{\dot{\omega}_{s}}\right)$ (Goldreich \& Peale 1966; Murray \& Dermott 2000). If capture does not occur, the satellite will spin up until it breaks apart. This would also be the satellite's fate if the radiative torque drives the spin away from synchroneity. But the story would not end here. Following breakup, the debris would again collect into a satellite and the process would repeat until synchroneity was achieved. ${ }^{12}$ Papers by Scheeres (2007) and Walsh et al. (2008) describe the fission of rapidly spinning rubble piles.

\subsection{Distribution of Binary Semimajor Axes}

Binaries evolving under BYORP pass through a logarithmic interval of semimajor axis on a timescale $a /(d a / d t) \propto a^{-1 / 2}$ (see Equation (33)). Thus the differential distribution of their semimajor axes should be proportional to $a^{-3 / 2}$. The distribution of semimajor axes of near-Earth binaries (see Table 1) appears to be compatible with an evolution dominated by BYORP. However, tidal torques may be required to account for the substantial fraction of the larger main-belt binaries having $a \geqslant 10 R_{p}$ (see data in Table 2).

\subsection{Eccentricity Evolution}

BYORP damps the binary's orbital eccentricity. Ćuk \& Burns (2005) show that the rate at which the eccentricity damps is proportional to that at which the semimajor axis increases. However, their derivation of the constant of proportionality is flawed. An improved derivation follows. For clarity we adopt a few inessential restrictions. We assume that the binary's center of mass moves on a circle around the Sun and that the binary's orbit lies in the plane defined by this circle. Moreover, we neglect the mass of the secondary with respect to that of the primary.

\footnotetext{
${ }^{10} f_{B Y}$ can be positive or negative.

11 This statement is valid for a circular orbit but might not be for an eccentric orbit.

12 We are assuming that there is adequate time for this to occur.
} 
Table 1

\begin{tabular}{|c|c|c|c|c|c|c|c|c|}
\hline $\begin{array}{l}\text { Asteroid } \\
\text { Name }\end{array}$ & $\begin{array}{r}\text { Period } \\
\text { (days) }\end{array}$ & $\begin{array}{c}a \\
(\mathrm{~km})\end{array}$ & $\begin{array}{c}\text { Primary } \\
\text { Diam. }(\mathrm{km})\end{array}$ & $\begin{array}{c}\text { Secondary } \\
\text { Diam. }(\mathrm{km})\end{array}$ & $\begin{array}{c}\text { Tidal Age } \\
(\mathrm{Myr})\end{array}$ & $\mathcal{R}_{\dot{a}}$ & $\begin{array}{l}\text { Spin-down } \\
\text { Time (kyr) }\end{array}$ & $\mathcal{R}_{\dot{\omega}_{s}}$ \\
\hline$(66391) 1999 \mathrm{KW}_{4}$ & 0.73 & 2.5 & 1.2 & 0.4 & 9.9 & 0.0089 & 110 & 6.8 \\
\hline $1999 \mathrm{HF}_{1}$ & 0.58 & 7.0 & 3.5 & 0.8 & 6.7 & 0.13 & 50 & 110 \\
\hline (5381) Sekhmet & 0.52 & 1.5 & 1.0 & 0.3 & 2.2 & 0.019 & 170 & 1.6 \\
\hline (66063) $1998 \mathrm{RO}_{1}$ & 0.6 & 1.4 & 0.8 & 0.38 & 1.8 & 0.025 & 130 & 4.0 \\
\hline $1996 \mathrm{FG}_{3}$ & 0.67 & 2.6 & 1.5 & 0.47 & 3.5 & 0.023 & 120 & 5.3 \\
\hline (88710) $2001 \mathrm{SL}_{9}$ & 0.68 & 1.4 & 0.8 & 0.22 & 10 & 0.0020 & 250 & 0.55 \\
\hline $1994 \mathrm{AW}_{1}$ & 0.93 & 2.3 & 1 & 0.5 & 7.6 & 0.0076 & 100 & 7.8 \\
\hline $2003 \mathrm{YT}_{1}$ & 1.2 & 2.7 & 1 & 0.18 & 460 & 0.000042 & 290 & 0.37 \\
\hline (35107) $1991 \mathrm{VH}$ & 1.4 & 3.2 & 1.2 & 0.44 & 48 & 0.00082 & 130 & 3.3 \\
\hline $2000 \mathrm{DP}_{107}$ & 1.8 & 2.6 & 0.8 & 0.3 & 230 & 0.000078 & 190 & 1.2 \\
\hline (65803) Didymos & 0.49 & 1.1 & 0.8 & 0.17 & 4.7 & 0.0034 & 330 & 0.23 \\
\hline (5407) $1992 \mathrm{AX}$ & 0.56 & 6.8 & 3.9 & 0.78 & 4.4 & 0.14 & 60 & 54 \\
\hline (85938) $1999 \mathrm{DJ}_{4}$ & 0.74 & 0.8 & 0.4 & 0.17 & 12 & 0.00080 & 290 & 0.36 \\
\hline $2000 \mathrm{UG}_{11}$ & 0.77 & 0.4 & 0.2 & 0.08 & 30 & 0.000068 & 640 & 0.033 \\
\hline (3671) Dionysus & 1.2 & 3.8 & 1.5 & 0.3 & 150 & 0.00031 & 180 & 1.6 \\
\hline $2002 \mathrm{CE}_{26}$ & 0.67 & 5.1 & 3 & 0.21 & 140 & 0.00050 & 260 & 0.45 \\
\hline
\end{tabular}

Notes. Characteristics of well-observed NEA binaries—Column 1: orbital period; Column 2: semimajor axis; Column 3: primary diameter; Column 4: secondary diameter; Column 5: calculated time to reach current semimajor axis based on tidal torque with $k_{2 p}$ from rubble pile model and $Q_{p}=100$; Column 6: ratio of rates of semimajor axis variation due to tides and BYORP at current semimajor axis based on $f_{\mathrm{BY}}=10^{-3}$; Column 7: calculated time for secondary to achieve synchronous rotation starting from maximal spin rate based on tidal torque with $k_{2 s}$ from rubble pile model and $Q_{s}=100$; Column 8: ratio of rates of spin variation due to tides and YORP at Roche radius with $f_{Y}=4 \times 10^{-4}$. Binary parameters were taken from a compilation by Walsh \& Richardson (2006).

Table 2

\begin{tabular}{|c|c|c|c|c|c|c|c|c|}
\hline $\begin{array}{l}\text { Primary Asteroid } \\
\text { Name }\end{array}$ & $\begin{array}{r}\text { Period } \\
\text { (days) }\end{array}$ & $\begin{array}{c}a \\
(\mathrm{~km})\end{array}$ & $\begin{array}{c}\text { Primary } \\
\text { Diam. }(\mathrm{km})\end{array}$ & $\begin{array}{l}\text { Secondary } \\
\text { Diam. (km) }\end{array}$ & $\begin{array}{l}\text { Tidal Age } \\
\text { (Gyr) }\end{array}$ & $\mathcal{R}_{\dot{a}}$ & $\begin{array}{l}\text { Spin-down } \\
\text { Time (kyr) }\end{array}$ & $\mathcal{R}_{\dot{\omega}_{s}}$ \\
\hline (22) Kalliope & 3.6 & 1100 & 180 & 26 & 0.85 & 5.6 & 1.7 & $1.9 \times 10^{7}$ \\
\hline (45) Eugenia & 4.8 & 1200 & 220 & 7 & 47 & 0.0077 & 9.7 & $5.3 \times 10^{4}$ \\
\hline (107) Camilla & 3.7 & 1300 & 240 & 16 & 3.2 & 0.88 & 3.5 & $2.2 \times 10^{6}$ \\
\hline (762) Pulcova & 4.4 & 700 & 140 & 19 & 0.61 & 1.26 & 3.8 & $1.1 \times 10^{6}$ \\
\hline (87) Sylvia & 1.4 & 710 & 290 & 7 & 0.45 & 3.0 & 9.3 & $1.0 \times 10^{5}$ \\
\hline (87) Sylvia & 3.7 & 1400 & 290 & 1.8 & 1800 & 0.00014 & 35.8 & $1.8 \times 10^{3}$ \\
\hline (243) Ida & 1.5 & 110 & 31 & 1.4 & 4.2 & 0.014 & 30.9 & $2.9 \times 10^{3}$ \\
\hline (130) Elektra & 3.9 & 1300 & 180 & 4 & 470 & 0.0022 & 9.9 & $1.1 \times 10^{5}$ \\
\hline (283) Emma & 3.4 & 600 & 150 & 12 & 0.68 & 0.67 & 6.3 & $2.1 \times 10^{5}$ \\
\hline (379) Huenna & 81 & 3400 & 92 & 7 & $2.1 \times 10^{6}$ & $2.4 \times 10^{7}$ & 9.4 & $8.0 \times 10^{4}$ \\
\hline
\end{tabular}

Notes. Characteristics of well-observed main-belt binaries—Column 1: orbital period; Column 2: semimajor axis; Column 3: primary diameter; Column 4: secondary diameter; Column 5: calculated time to reach current semimajor axis based on tidal torque with $k_{2 p}$ from rubble pile model and $Q_{p}=100$; Column 6: ratio of rates of semimajor axis variation due to tides and BYORP at current semimajor axis based on $f_{\mathrm{BY}}=10^{-3}$; Column 7: calculated time for secondary to achieve synchronous rotation starting from maximal spin rate based on tidal torque with $k_{2 s}$ from rubble pile model and $Q_{s}=100$; Column 8: ratio of rates of spin variation due to tides and YORP at Roche radius with $f_{Y}=4 \times 10^{-4}$. Parameters for the binaries were taken from http://www.johnstonsarchive.net/astro/asteroidmoons.html\#2 as well as Marchis et al. (2008).

Two essential requirements are that the secondary's rate of spin match the orbit's mean motion,,$^{13}$ and that the angle between the binary's apsidal line and the direction to the Sun is random. ${ }^{14}$ This allows the secular evolution of the binary's orbit under BYORP to be evaluated by considering the Sun to be uniformly spread around the binary for a fixed orientation of its apsidal line. Given these conditions, the net radiation force per unit mass acting on the secondary, $\vec{F}$, has fixed magnitude and rotates uniformly with the body. It can be decomposed into components aligned with the apsidal axis, $F_{x}=F \cos (\lambda+\theta)$, and orthogonal to the apsidal axis, $F_{y}=F \sin (\lambda+\theta)$. To first

\footnotetext{
13 Forced librations can be neglected.

${ }^{14}$ Precession of the binary's apsidal line is independent of the position of the sun.
}

order in eccentricity, $e$, the mean anomaly, $\lambda$, is related to the true anomaly, $f$, by $\lambda \approx f-2 e \sin f$.

To zeroth order in $e$, semimajor axis evolution depends upon the tangential component of $\vec{F}, T \equiv-\sin f F_{x}+\cos f F_{y}=$ $F \sin (\lambda-f+\theta) \approx F \sin \theta$. A straightforward calculation yields

$$
\frac{1}{a} \frac{d a}{d t}=\frac{2 F}{n a} \sin \theta
$$

Eccentricity evolution is examined with the aid of the eccentricity vector ${ }^{15}$

$$
\vec{e} \equiv \frac{\vec{v} \times(\vec{r} \times \vec{v})}{n^{2} a^{3}}-\hat{r}
$$

\footnotetext{
${ }^{15}$ Here $\hat{r} \equiv \vec{r} / r$, which differs from its meaning in Section 2 .
} 
Under the action of $\vec{F}, \vec{e}$ evolves according to

$$
\dot{\vec{e}}=\frac{\vec{F} \times(\vec{r} \times \vec{v})+\vec{v} \times(\vec{r} \times \vec{F})}{n^{2} a^{3}} .
$$

Since angular momentum is a constant of motion and $\vec{F}$ rotates uniformly, the first term is periodic and averages to zero. Projecting the second term along $\hat{x}$, we arrive at

$$
\dot{e}=\frac{v_{y}\left(x F_{y}-y F_{x}\right)}{n^{2} a^{3}}
$$

where to the first order in eccentricity $x=(1-e \cos f) \cos f$, $y=(1-e \cos f) \sin f$, and $v_{y}=(\cos f-e \cos 2 f) \dot{f}$. Averaging Equation (38) over a period of the binary orbit yields

$$
\frac{1}{e} \frac{d e}{d t}=-\frac{F}{2 n a} \sin \theta
$$

Dividing Equation (39) by Equation (35), we obtain

$$
\frac{1}{e} \frac{d e}{d t}=-\frac{1}{4 a} \frac{d a}{d t}
$$

from which it follows that $e \propto a^{-1 / 4}$.

Our expression for $d e / d t$ is a factor of 3 smaller than that given by Ćuk \& Burns (2005). Their error was to assume that the radial and tangential components of $\vec{F}$ are constant in time. Since the secondary rotates uniformly, while its true anomaly does not, this assertion is incorrect. A short calculation suffices to show that to the first order in $e$

$$
\begin{aligned}
& R=F \cos (\lambda-f+\theta) \approx F(\cos \theta+2 e \sin \theta \sin \lambda) \\
& T=F \sin (\lambda-f+\theta) \approx F(\sin \theta-2 e \cos \theta \sin \lambda) .
\end{aligned}
$$

It is the term in $R$ of first order in $e$ that is responsible for the difference between our expression for $d e / d t$ and that obtained by Ćuk \& Burns (2005).

The weak dependence of eccentricity on semimajor axis makes eccentricity damping by BYORP irrelevant in all circumstances. In the special case that the effect of tidal and radiative torques on semimajor axis evolution cancel, tides would dominate eccentricity evolution.

\subsection{Application to Binary Near-Earth Asteroids}

Table 1 provides data relevant for the comparison of tidal and radiative evolution rates for binary near-Earth asteroids. Despite substantial uncertainties in appropriate choices for $Q_{p}$, $Q_{s}, f_{Y}$, and $f_{B Y}$, some general inferences can be drawn from these entries.

It is apparent that BYORP dominates tidal orbit expansion at the current values of semimajor axis. It is less certain, but plausible, that tides were responsible for synchronizing the spins of the secondaries. That the tidal torque is even remotely competitive with the radiative torque is due to the large (3-4 orders of magnitude) enhancement of Love number for a rubble pile with a radius of a fraction of a kilometer. Synchronization is most problematic for small secondaries orbiting small primaries. However, as described in Section 5.3, a small secondary that fails to achieve synchronization spins up to where it breaks apart and re-forms. This scenario implies that small secondaries may achieve synchronization with unusually low values of $f_{Y}$.
Direct evidence favoring radiation torques over tidal torques in the expansion of near-earth asteroid (NEA) binaries comes from the observation that the primary of the NEA binary KW4 is spinning at close to its disruption threshold. This would be puzzling if the binary had conserved its total angular momentum since the secondary separated from the original primary. Such would be the case if tides were responsible for the orbit's expansion. The puzzle is resolved if the YORP torque spun up the original primary causing it to fission, and then either BYORP or tides drove the orbit expansion (Scheeres et al. 2006). In the latter case, YORP could replace the angular momentum transferred from the spin of the primary to the orbit of the binary.

\subsection{Application to Binary Main-Belt Asteroids}

Table 2 contains the same information for main-belt binary asteroids as Table 1 does for binary NEAs. The dominance of the tidal torque over the radiative torque in spin synchronization of the satellite at the Roche radius is evident. For most binaries it would be apparent even without the substantial (about 2 orders of magnitude) enhancement of the tidal Love number for a rubble pile.

No general conclusion can be drawn about the relative importance of tidal and radiative torques in driving the evolution of semimajor axis. For some systems the radiative torque obviously dominates and their orbits must be expanding. In others, an equilibrium might exist in which angular momentum added to the orbit by the tidal torque is removed by the radiative torque.

This research was supported in part by an NSF grant, a NASA grant, and an ERC grant. R.S. is an Alfred P. Sloan Fellow and a Packard Fellow. We thank Hiroo Kanamori for valuable advice. We are particularly grateful to the anonymous referee who alerted us to the binary YORP effect. Our views concerning the evolution of binary asteroids underwent a major revision as a result of the referee's suggestions.

\section{REFERENCES}

Ćuk, M., \& Burns, J. A. 2005, Icarus, 176, 418

Duffy, J., \& Mindlin, R. D. 1957, J. Appl. Mech., 24, 585

Goddard, J. D. 1990, Proc. Math. Phys. Sci., 430, 105

Goldreich, P. 1963, MNRAS, 126, 257

Goldreich, P., \& Peale, S. 1966, AJ, 71, 425

Goldreich, P., \& Soter, S. 1966, Icarus, 5, 375

Jacobson, R. A., \& French, R. G. 2004, Icarus, 172, 382

Lowry, S. C., et al. 2007, Science, 316, 272

Marchis, F., Descamps, P., Baek, M., Harris, A. W., Kaasalainen, M., Berthier, J., D., Hestroffer, \& F., Vachier 2008, arXiv:0804.1383v1

Margot, J. L., \& Brown, M. E. 2003, Science, 300, 1939

Murray, C. D., \& Dermott, S. F. 2000, Solar System Dynamics (Cambridge: Cambridge Univ. Press)

Ostro, S. J., et al. 2006, Science, 314, 1276

Porco, C. C., Thomas, P. C., W., W. J., \& Richardson, D. C. 2007, Science, 318, 1602

Rubincam, D. P. 2000, Icarus, 148, 2

Scheeres, D. J. 2007, Icarus, 189, 370

Scheeres, D. J., et al. 2006, Science, 314, 1280

Taylor, P. A., et al. 2007, Science, 316, 274

Veverka, J., et al. 1997, Science, 278, 2109

Walsh, K. J., \& Richardson, D. C. 2006, Icarus, 180, 201

Walsh, K. J., Richardson, D. C., \& Michel, P. 2008, Nature, 454, 188

Williams, J. G., Boggs, D. H., \& Ratcliff, J. T. 2008, Lunar and Planetary Institute Conference Abstracts, 39, 1484

Yeomans, D. K., et al. 1997, Science, 278, 2106 